\title{
STRONG OPTIMALITY OF BOLD PLAY FOR DISCOUNTED DUBINS-SAVAGE GAMBLING PROBLEMS WITH TIME-DEPENDENT PARAMETERS
}

\author{
YI-CHING YAO, ${ }^{* *}$ Academia Sinica and National Chengchi University \\ MAY-RU CHEN, ${ }^{* * * *}$ Academia Sinica
}

\begin{abstract}
In the classic Dubins-Savage subfair primitive casino gambling problem, the gambler can stake any amount in his possession, winning $(1-r) / r$ times the stake with probability $w$ and losing the stake with probability $1-w, 0 \leq w \leq r \leq 1$. The gambler seeks to maximize the probability of reaching a fixed fortune (the goal) by gambling repeatedly with suitably chosen stakes. This problem has recently been extended in a unifying framework to account for limited playing time as well as future discounting, under which bold play is known to be optimal provided that $w \leq \frac{1}{2} \leq r$. This paper is concerned with a further extension of the Dubins-Savage gambling problem involving time-dependent parameters, and shows that bold play not only maximizes the probability of reaching the goal, but also stochastically minimizes the number of plays needed to reach the goal. As a result, bold play also maximizes the expected utility, where the utility at the goal is only required to be monotone decreasing with respect to the number of plays needed to reach the goal. It is further noted that bold play remains optimal even when the time-dependent parameters are random.
\end{abstract}

Keywords: Gambling theory; primitive casino; discount factor; optimal strategy

2000 Mathematics Subject Classification: Primary 60G40

Secondary $91 \mathrm{~A} 60$

\section{Introduction}

In the classic Dubins-Savage primitive casino gambling problem with parameters $0<r \leq 1$ and $0 \leq w \leq 1$, the gambler can stake any amount in his possession, winning $\bar{r} / r$ times the stake with probability $w$ and losing the stake with probability $\bar{w}$, where $\bar{r}=1-r$ and $\bar{w}=1-w$. The gambler seeks to maximize the probability of reaching a fixed fortune (to be normalized to unity) by gambling repeatedly with suitably chosen stakes. In an ingenious proof, Dubins and Savage [5, Chapter 6] showed that, for the subfair case, in which $w \leq r$, the maximum probability of reaching fortune 1 (the goal) is attained by the bold strategy 'staking on each play as much as possible without risk of overshooting the goal', i.e. staking $\min \{f,(1-f) r / \bar{r}\}$ if the current fortune is $0<f<1$. When the gambler is allowed to play at most $n$ times $(1 \leq n<\infty)$, Dvoretzky proved (see [5, pp. 92-95]) that bold play remains optimal provided that either $w \leq \frac{1}{2}=r$ (the subfair 'red-and-black' casino setting) or $w=\frac{1}{2} \leq r$ (the 'taxedcoin' casino setting).

Received 30 August 2007; revision received 23 April 2008.

* Postal address: Institute of Statistical Science, Academia Sinica, Taipei 115, Taiwan, R.O.C.

** Email address: yao@stat.sinica.edu.tw

*** Email address: mayru@stat.sinica.edu.tw 
Chen [2] introduced an inflation-discounted version of the Dubins-Savage gambling problem in which the gambler's fortune is discounted after each play by a factor of $\alpha, 0 \leq \alpha \leq 1$, while the objective remains to maximize the probability of reaching (discounted) fortune 1 . Note that $\bar{\alpha} / \alpha=(1-\alpha) / \alpha \geq 0$ may be interpreted as the interest or inflation rate, where $1 / 0$ is defined as $\infty$. For this inflation-discounted version, if the gambler stakes an amount $s$ of his fortune $f$ then his fortune becomes $\alpha(f+s \bar{r} / r)$ with probability $w$ and $\alpha(f-s)$ with probability $\bar{w}$. To play boldly is to stake $\min \left\{f,\left(\alpha^{-1}-f\right) r / \bar{r}\right\}$ when the current fortune is $0<f<1$. Chen [2] proved that the bold strategy is optimal if $w \leq \frac{1}{2}=r$, which has been improved to the more general condition $w \leq \frac{1}{2} \leq r$ by Chen et al. [4]. It was further extended in [15] to the finite-horizon setting and shown that when the gambler is allowed to play at most $n$ times $(1 \leq n<\infty)$, the bold strategy remains optimal provided that $w \leq \frac{1}{2} \leq r$. It should be noted that the bold strategy depends on the inflation-discount factor $\alpha$, but not on the finite horizon $n$.

Let $\tau_{B}$ and $\tau_{S}$ respectively denote the number of plays needed for the bold strategy and an arbitrary strategy $S$ to reach the goal. Note that $\tau_{B}$ and $\tau_{S}$ are extended-valued stopping times such that $\left\{\tau_{B}<\infty\right\}$ and $\left\{\tau_{S}<\infty\right\}$ are the events that the bold strategy and strategy $S$, respectively, eventually reach the goal. While Chen et al. [4] showed that $\mathrm{P}\left(\tau_{B}<\infty\right) \geq$ $\mathrm{P}\left(\tau_{S}<\infty\right)$, it follows from [15] that, for each $n=1,2, \ldots, \mathrm{P}\left(\tau_{B} \leq n\right) \geq \mathrm{P}\left(\tau_{S} \leq n\right)$, implying that $\tau_{B}$ is stochastically smaller than $\tau_{S}$. In other words, the bold strategy not only maximizes the probability of reaching the goal, but also stochastically minimizes the number of plays needed to reach the goal. (This latter stochastic minimization property of the bold strategy is not explicitly pointed out in [15].)

This paper is concerned with a further extension of the Dubins-Savage gambling problem involving time-dependent parameters. Specifically, given the sequences $\boldsymbol{r}=\left(r_{1}, r_{2}, \ldots\right), \boldsymbol{w}=$ $\left(w_{1}, w_{2}, \ldots\right)$, and $\boldsymbol{\alpha}=\left(\alpha_{1}, \alpha_{2}, \ldots\right)$, if the gambler stakes an amount $s$ of his fortune $f$ on the $k$ th play then his fortune becomes $\alpha_{k}\left(f+s \bar{r}_{k} / r_{k}\right)$ with probability $w_{k}$ and $\alpha_{k}(f-s)$ with probability $\bar{w}_{k}$, where $\bar{r}_{k}=1-r_{k}$ and $\bar{w}_{k}=1-w_{k}$. The objective is to maximize the probability of reaching fortune 1 in $n$ plays $(1 \leq n \leq \infty)$. (Here $n=\infty$ refers to the infinite-horizon setting.) To play boldly on the $k$ th play with fortune $0<f<1$ is to stake $\min \left\{f,\left(\alpha_{k}^{-1}-f\right) r_{k} / \bar{r}_{k}\right\}$, which depends on $r_{k}$ and $\alpha_{k}$. In the next section it is shown (Theorem 1) that under the condition

$$
0 \leq \cdots \leq w_{2} \leq w_{1} \leq \frac{1}{2} \leq r_{1} \leq r_{2} \leq \cdots \leq 1 \quad \text { and } \quad 0 \leq \alpha_{k} \leq 1 \quad \text { for all } k,
$$

the bold strategy both maximizes the probability of reaching the goal and stochastically minimizes the number of plays needed to reach the goal. This result includes [15, Theorem 1] as a special case. It is also noted (Remark 2) that the bold strategy maximizes the expected utility, where the utility at the goal is only required to be monotone decreasing with respect to the number of plays needed to reach the goal. Furthermore, it is observed (Remark 3 ) that the bold strategy remains optimal even when the time-dependent parameters are random. Section 3 contains technical proofs. We close this section by mentioning that results on the optimality of the bold strategy for continuous-time gambling problems can be found in [14] and recent works on related problems can be found in [1], [6], [9], [10], [11], [13], and [16].

\section{Main results}

Write $\boldsymbol{H}=(\boldsymbol{r}, \boldsymbol{w}, \boldsymbol{\alpha})$, which specifies the time-dependent parameter values, and let $Q_{n}(f ; \boldsymbol{H}), 0 \leq n \leq \infty$, denote the probability of reaching the goal in $n$ plays under the bold strategy (with initial fortune $f)$. Here $Q_{\infty}(f ; \boldsymbol{H})$ is the probability of attaining the goal when the gambler uses the bold strategy with unlimited playing time. Note that $Q_{0}(f ; \boldsymbol{H})=$ 
$\mathbf{1}_{[1, \infty)}(f)$, where $\mathbf{1}_{A}$ denotes the indicator function of a set $A$, and that $Q_{n}(f ; \boldsymbol{H})$ depends on $\boldsymbol{H}=(\boldsymbol{r}, \boldsymbol{w}, \boldsymbol{\alpha})$ only through the first $n$ components of each of $\boldsymbol{r}, \boldsymbol{w}$, and $\boldsymbol{\alpha}$. By conditioning on the first play, we have, for $0<\alpha_{1} \leq 1$ and $n=0,1, \ldots$,

$$
Q_{n+1}(f ; \boldsymbol{H})= \begin{cases}w_{1} Q_{n}\left(\frac{\alpha_{1} f}{r_{1}} ; \boldsymbol{H}^{(1)}\right) & \text { if } 0 \leq f<\min \left\{r_{1} / \alpha_{1}, 1\right\}, \\ \bar{w}_{1} Q_{n}\left(\frac{f-r_{1} / \alpha_{1}}{\bar{r}_{1} / \alpha_{1}} ; \boldsymbol{H}^{(1)}\right)+w_{1} & \text { if } \min \left\{r_{1} / \alpha_{1}, 1\right\} \leq f<1, \\ 1 & \text { if } f \geq 1,\end{cases}
$$

where $\boldsymbol{H}^{(k)}=\left(\boldsymbol{r}^{(k)}, \boldsymbol{w}^{(k)}, \boldsymbol{\alpha}^{(k)}\right), \boldsymbol{r}^{(k)}=\left(r_{k+1}, r_{k+2}, \ldots\right), \boldsymbol{w}^{(k)}=\left(w_{k+1}, w_{k+2}, \ldots\right)$, and $\boldsymbol{\alpha}^{(k)}=\left(\alpha_{k+1}, \alpha_{k+2}, \ldots\right), k=1,2, \ldots$

To prove (2), suppose that the gambler is allowed to play at most $n+1$ times with the timedependent parameter vector $\boldsymbol{H}=(\boldsymbol{r}, \boldsymbol{w}, \boldsymbol{\alpha})$. If his initial fortune is $0 \leq f<\min \left\{r_{1} / \alpha_{1}, 1\right\}$ then, under the bold strategy, he would stake all of his fortune on the first play. He either goes broke (with probability $\bar{w}_{1}$ ) or has a fortune of $\alpha_{1} f / r_{1}<1$ (with probability $w_{1}$ ). In the latter case, he can still play up to $n$ times for which the relevant time-dependent parameter values are $\left(r_{2}, \ldots, r_{n+1}\right),\left(w_{2}, \ldots, w_{n+1}\right)$, and $\left(\alpha_{2}, \ldots, \alpha_{n+1}\right)$. In other words, upon winning the first bet, the gambler faces a new situation with (current) fortune $\alpha_{1} f / r_{1}$, horizon $n$, and timedependent parameter vector $\boldsymbol{H}^{(1)}$ replacing, respectively, (original) $f, n+1$, and $\boldsymbol{H}$. So, given that the gambler wins the first bet, the conditional probability that the gambler, using the bold strategy, reaches the goal (in at most $n$ more plays) equals $Q_{n}\left(\alpha_{1} f / r_{1} ; \boldsymbol{H}^{(1)}\right)$. It follows that $Q_{n+1}(f ; \boldsymbol{H})=w_{1} Q_{n}\left(\alpha_{1} f / r_{1} ; \boldsymbol{H}^{(1)}\right)$ if $0 \leq f<\min \left\{r_{1} / \alpha_{1}, 1\right\}$. If $\min \left\{r_{1} / \alpha_{1}, 1\right\} \leq f<1$ (which is not vacuous only when $r_{1} / \alpha_{1}<1$ ) then, under the bold strategy, the gambler stakes an amount $s=\left(\alpha_{1}^{-1}-f\right) r_{1} / \bar{r}_{1}$ of his fortune $f$ on the first play. He either wins the bet to reach the goal (with probability $\left.w_{1}\right)$ or loses the bet with a remaining fortune of $\left(\alpha_{1} f-r_{1}\right) / \bar{r}_{1}$ (with probability $\bar{w}_{1}$ ). In the latter case the gambler faces a new situation with (current) fortune $\left(\alpha_{1} f-r_{1}\right) / \bar{r}_{1}$, horizon $n$, and time-dependent parameter vector $\boldsymbol{H}^{(1)}$ replacing, respectively, (original) $f, n+1$, and $\boldsymbol{H}$. So, given that the gambler loses the first bet, the conditional probability that the gambler, using the bold strategy, reaches the goal (in at most $n$ more plays) equals $Q_{n}\left(\left(\alpha_{1} f-r_{1}\right) / \bar{r}_{1} ; \boldsymbol{H}^{(1)}\right)$. It follows that $Q_{n+1}(f ; \boldsymbol{H})=\bar{w}_{1} Q_{n}\left(\left(\alpha_{1} f-r_{1}\right) / \bar{r}_{1} ; \boldsymbol{H}^{(1)}\right)+$ $w_{1}$ if $\min \left\{r_{1} / \alpha_{1}, 1\right\} \leq f<1$. This proves (2). Furthermore, it is readily seen that, as $n \rightarrow \infty$, $Q_{n}(f ; \boldsymbol{H})$ increases to $Q_{\infty}(f ; \boldsymbol{H})$.

Next, let $U_{n}(f ; \boldsymbol{H})$ denote the maximum probability of attaining the goal with initial fortune $f$ when the gambler is allowed to play at most $n$ times $(n=0,1,2, \ldots)$, and let $U_{\infty}(f ; \boldsymbol{H})$ be the maximum probability of attaining the goal with initial fortune $f$ when there is unlimited playing time. Then $U_{0}(f ; \boldsymbol{H})=\mathbf{1}_{[1, \infty)}(f), f \geq 0$. By conditioning on the first play, we have, for $n=0,1, \ldots$,

$$
U_{n+1}(f ; \boldsymbol{H})=\left\{\begin{aligned}
\sup \left\{\bar{w}_{1} U_{n}\left(\alpha_{1}(f-y) ; \boldsymbol{H}^{(1)}\right)\right. & \\
\left.+w_{1} U_{n}\left(\alpha_{1}\left(f+\frac{y \bar{r}_{1}}{r_{1}}\right) ; \boldsymbol{H}^{(1)}\right): 0 \leq y \leq f\right\} & \text { if } 0 \leq f<1, \\
1 & \text { if } f \geq 1 .
\end{aligned}\right.
$$

To prove (3), note that, for each $y$ with $0 \leq y \leq f<1, \bar{w}_{1} U_{n}\left(\alpha_{1}(f-y) ; \boldsymbol{H}^{(1)}\right)+w_{1} U_{n}\left(\alpha_{1}(f+\right.$ $\left.\left.y \bar{r}_{1} / r_{1}\right) ; \boldsymbol{H}^{(1)}\right)$ is the probability of reaching the goal when the gambler stakes the amount $y$ 
out of his fortune $f$ on the first play and then plays optimally in the next (up to) $n$ plays. It follows that the maximum value of this probability over $0 \leq y \leq f$ must equal $U_{n+1}(f ; \boldsymbol{H})$, the maximum probability of reaching the goal in $n+1$ plays. This proves (3). Furthermore, as $n \rightarrow \infty, U_{n}(f ; \boldsymbol{H})$ increases to $U_{\infty}(f ; \boldsymbol{H})$ (cf. [5, Section 2.15] and [8, Section 3.6]).

Theorem 1. If $\boldsymbol{H}=(\boldsymbol{r}, \boldsymbol{w}, \boldsymbol{\alpha})$ satisfies condition (1) then the maximum probability of reaching the goal in $n$ plays $(1 \leq n \leq \infty)$ is attained by the bold strategy, i.e. $Q_{n}(f ; \boldsymbol{H})=U_{n}(f ; \boldsymbol{H})$ for $f \geq 0$ and $n=0,1, \ldots, \infty$.

Proof. It follows from (2) and Proposition 1, below, that, for $n=0,1, \ldots$,

$$
Q_{n+1}(f ; \boldsymbol{H})=\left\{\begin{aligned}
\sup \left\{\bar{w}_{1} Q_{n}\left(\alpha_{1}(f-y) ; \boldsymbol{H}^{(1)}\right)\right. & \\
\left.+w_{1} Q_{n}\left(\alpha_{1}\left(f+\frac{y \bar{r}_{1}}{r_{1}}\right) ; \boldsymbol{H}^{(1)}\right): 0 \leq y \leq f\right\} & \text { if } 0 \leq f<1, \\
1 & \text { if } f \geq 1 .
\end{aligned}\right.
$$

In view of (3), the two sequences of functions $\left\{Q_{n}(f ; \boldsymbol{H}), n=0,1, \ldots\right\}$ and $\left\{U_{n}(f ; \boldsymbol{H})\right.$, $n=0,1, \ldots\}$ satisfy the same recursion equation with the same initial condition $Q_{0}(f ; \boldsymbol{H})=$ $U_{0}(f ; \boldsymbol{H})=\mathbf{1}_{[1, \infty]}(f)$. Note that if $\boldsymbol{H}$ satisfies condition (1), so does $\boldsymbol{H}^{(1)}$. By induction, for all $\boldsymbol{H}$ satisfying condition (1) and all $f \geq 0$, we have $Q_{n}(f ; \boldsymbol{H})=U_{n}(f ; \boldsymbol{H})$ for $n=0,1, \ldots$ and $Q_{\infty}(f ; \boldsymbol{H})=\lim _{n \rightarrow \infty} Q_{n}(f ; \boldsymbol{H})=\lim _{n \rightarrow \infty} U_{n}(f ; \boldsymbol{H})=U_{\infty}(f ; \boldsymbol{H})$. The proof is complete.

Proposition 1. Assume that $\boldsymbol{H}=(\boldsymbol{r}, \boldsymbol{w}, \boldsymbol{\alpha})$ satisfies condition (1). Then, for $n=0,1, \ldots$ and $0 \leq y \leq f<1$,

$$
Q_{n+1}(f ; \boldsymbol{H}) \geq \bar{w}_{1} Q_{n}\left(\alpha_{1}(f-y) ; \boldsymbol{H}^{(1)}\right)+w_{1} Q_{n}\left(\alpha_{1}\left(f+\frac{y \bar{r}_{1}}{r_{1}}\right) ; \boldsymbol{H}^{(1)}\right) .
$$

The proof of Proposition 1 is given in Section 3. A few remarks are in order.

Remark 1. Condition (1) requires the pair $\left(r_{k}, w_{k}\right)$ to be less favorable to the gambler for larger $k$. However, the 'inflation-discount' sequence $\boldsymbol{\alpha}$ is not required to be monotone.

Remark 2. Suppose that $\boldsymbol{H}=(\boldsymbol{r}, \boldsymbol{w}, \boldsymbol{\alpha})$ satisfies (1). Let $\tau_{B}$ and $\tau_{S}$ respectively denote the number of plays needed for the bold strategy and an arbitrary strategy $S$ to reach the goal. It follows from Theorem 1 that

$$
\mathrm{P}\left(\tau_{B} \leq n\right) \geq \mathrm{P}\left(\tau_{S} \leq n\right), \quad n=1,2, \ldots, \quad \text { and } \quad \mathrm{P}\left(\tau_{B}<\infty\right) \geq \mathrm{P}\left(\tau_{S}<\infty\right),
$$

implying that $\tau_{B}$ is stochastically smaller than $\tau_{S}$ (i.e. the bold strategy stochastically minimizes the number of plays needed to reach the goal). By a standard result on stochastic ordering (see, e.g. [12, p. 4]), we have $\mathrm{E} \beta\left(\tau_{B}\right) \geq \mathrm{E} \beta\left(\tau_{S}\right)$ for any monotone decreasing sequence $\beta(1) \geq \beta(2) \geq \cdots \geq \beta(\infty)>-\infty$. Here $\beta(k), k=1,2, \ldots$, may be interpreted as the utility received by the gambler if the goal is reached on the $k$ th play, and the sequence $\{\beta(k), k=1,2, \ldots, \infty\}$ is referred to as a utility-discount sequence. Hence, the bold strategy maximizes the expected utility with respect to any utility-discount sequence which is monotone decreasing. Note that the sequence $\beta(k)=1$ for $1 \leq k \leq n$ and $\beta(k)=0$ for $n+1 \leq k \leq \infty$ corresponds to the finite-horizon setting, where the gambler is allowed to play at most $n$ times, and that the sequence $\beta(k)=\rho^{k}, 1 \leq k \leq \infty$, for some $0<\rho<1$ is the geometric discount setting which was first studied by Klugman [7] and later by Chen and Zame [3]. 
Remark 3. To execute the $k$ th play under the bold strategy, all the gambler needs to know is the current fortune and the values of $r_{k}$ and $\alpha_{k}$. No knowledge of the later values of the parameters is needed. So the bold strategy is in fact optimal in the more general stochastic setting where $\boldsymbol{H}=(\boldsymbol{r}, \boldsymbol{w}, \boldsymbol{\alpha})$ is random, satisfying condition (1) with probability 1 , and $\left(r_{k}, \alpha_{k}\right)$ is unavailable to the gambler until the $k$ th play.

\section{Proofs of Proposition 1 and some auxiliary lemmas}

The following lemmas are needed in order to prove Proposition 1. Both Lemmas 1 and 2 follow from (2) easily.

Lemma 1. For $n=0,1, \ldots, Q_{n}(f ; \boldsymbol{H})$ is nondecreasing in $f$.

Lemma 2. For given $\boldsymbol{H}=(\boldsymbol{r}, \boldsymbol{w}, \boldsymbol{\alpha})$, let $\hat{\boldsymbol{H}}=(\boldsymbol{r}, \boldsymbol{w}, \hat{\boldsymbol{\alpha}})$, where $\hat{\boldsymbol{\alpha}}=\left(\hat{\alpha}_{1}, \alpha_{2}, \alpha_{3}, \ldots\right)$ with $0<\alpha_{1} \leq \hat{\alpha}_{1} \leq 1$. Then we have

$$
Q_{n}(f ; \boldsymbol{H})=Q_{n}\left(\frac{\alpha_{1} f}{\hat{\alpha}_{1}} ; \hat{\boldsymbol{H}}\right)
$$

for $n=0,1, \ldots$ and $0 \leq f<1$.

Lemma 3. Assume that $0<\alpha_{1} \leq 1$ and $r_{1}<1$. Then (4) holds for $\left(\alpha_{1}^{-1}-f\right) r_{1} / \bar{r}_{1} \leq y \leq$ $f<1$.

Proof. The lemma holds trivially (vacuously) if $\left(\alpha_{1}^{-1}-f\right) r_{1} / \bar{r}_{1}>f$ (i.e. $\left.f<r_{1} / \alpha_{1}\right)$. Consider the case in which $\left(\alpha_{1}^{-1}-f\right) r_{1} / \bar{r}_{1} \leq f<1$ (i.e. $\left.1>f \geq r_{1} / \alpha_{1}\right)$, and let

$$
B(f):=\min \left\{f, \frac{\left(\alpha_{1}^{-1}-f\right) r_{1}}{\bar{r}_{1}}\right\}=\frac{\left(\alpha_{1}^{-1}-f\right) r_{1}}{\bar{r}_{1}} .
$$

Then, for $B(f) \leq y \leq f$, we have

$$
\begin{aligned}
Q_{n+1}(f ; \boldsymbol{H}) & =\bar{w}_{1} Q_{n}\left(\alpha_{1}(f-B(f)) ; \boldsymbol{H}^{(1)}\right)+w_{1} \\
& \geq \bar{w}_{1} Q_{n}\left(\alpha_{1}(f-y) ; \boldsymbol{H}^{(1)}\right)+w_{1} Q_{n}\left(\alpha_{1}\left(f+\frac{y \bar{r}_{1}}{r_{1}}\right) ; \boldsymbol{H}^{(1)}\right),
\end{aligned}
$$

by (2), Lemma 1 , and the fact that $Q_{n}\left(x ; \boldsymbol{H}^{(1)}\right)=1$ for $x \geq 1$, completing the proof.

Lemma 4. Fix $m \geq 1$, and let $\boldsymbol{H}_{1}=(\boldsymbol{r}, \boldsymbol{w}, \boldsymbol{\alpha})$ be fixed and satisfy (1), where $0<\alpha_{1} \leq 1$, $0<\alpha_{2} \leq 1,0<w_{2} \leq w_{1}$, and $r_{1} \leq r_{2}<1$. Consider the following statements.

(S1) Inequality (4) holds for $n=m, \boldsymbol{H}=\boldsymbol{H}_{1}$, and all $0 \leq y \leq f<1$.

(S2) Inequality (4) holds for $n=m, \boldsymbol{H}=\boldsymbol{H}_{2}:=(\boldsymbol{r}, \boldsymbol{w}, \overline{\boldsymbol{\alpha}})$, and all $0 \leq y \leq f<1$, where $\overline{\boldsymbol{\alpha}}=\left(1, \alpha_{2}, \alpha_{3}, \ldots\right)$.

(S3) Inequality (4) holds for $n=m, \boldsymbol{H}=\boldsymbol{H}_{3}:=(\boldsymbol{r}, \boldsymbol{w}, \widetilde{\boldsymbol{\alpha}})$, and all $0 \leq y \leq f<1$, where $\tilde{\boldsymbol{\alpha}}=\left(1,1, \alpha_{3}, \alpha_{4}, \ldots\right)$.

(S4) Inequality (4) holds for $n=m, \boldsymbol{H}=\boldsymbol{H}_{4}:=(\boldsymbol{r}, \widetilde{\boldsymbol{w}}, \widetilde{\boldsymbol{\alpha}})$, and all $0 \leq y \leq f<1$, where $\widetilde{\boldsymbol{w}}=\left(w_{2}, w_{2}, w_{3}, w_{4}, \ldots\right)$.

(S5) Inequality (4) holds for $n=m, \boldsymbol{H}=\boldsymbol{H}_{5}:=(\tilde{\boldsymbol{r}}, \tilde{\boldsymbol{w}}, \widetilde{\boldsymbol{\alpha}})$, and all $0 \leq y \leq f<1$, where $\tilde{\boldsymbol{r}}=\left(r_{2}, r_{2}, r_{3}, r_{4}, \ldots\right)$. 
Then $(S 5) \Rightarrow(S 4) \Rightarrow(S 3) \Rightarrow(S 2) \Rightarrow(S 1)$, where $A \Rightarrow B$ signifies that statement $A$ implies statement $B$.

Proof. (i) $(S 2) \Rightarrow(S 1)$ : For any $0 \leq y \leq f<1$, we have

$$
\begin{aligned}
Q_{m+1}\left(f ; \boldsymbol{H}_{1}\right) & =Q_{m+1}\left(\alpha_{1} f ; \boldsymbol{H}_{2}\right) \\
& \geq \bar{w}_{1} Q_{m}\left(\alpha_{1} f-\alpha_{1} y ; \boldsymbol{H}_{2}^{(1)}\right)+w_{1} Q_{m}\left(\alpha_{1} f+\frac{\alpha_{1} y \overline{r_{1}}}{r_{1}} ; \boldsymbol{H}_{2}^{(1)}\right) \\
& =\bar{w}_{1} Q_{m}\left(\alpha_{1}(f-y) ; \boldsymbol{H}_{1}^{(1)}\right)+w_{1} Q_{m}\left(\alpha_{1}\left(f+\frac{y \overline{r_{1}}}{r_{1}}\right) ; \boldsymbol{H}_{1}^{(1)}\right),
\end{aligned}
$$

where the first equality follows from Lemma 2 , the inequality follows from statement (S2), and the last equality follows from $\boldsymbol{H}_{1}^{(1)}=\boldsymbol{H}_{2}^{(1)}$.

(ii) $(S 3) \Rightarrow(S 2)$ : By (2) and Lemma 2, we have, for $0 \leq f<r_{1}$,

$$
Q_{m+1}\left(f ; \boldsymbol{H}_{2}\right)=w_{1} Q_{m}\left(\frac{f}{r_{1}} ; \boldsymbol{H}_{2}^{(1)}\right)=w_{1} Q_{m}\left(\frac{\alpha_{2} f}{r_{1}} ; \boldsymbol{H}_{3}^{(1)}\right),
$$

and, for $r_{1} \leq f<1$,

$$
Q_{m+1}\left(f ; \boldsymbol{H}_{2}\right)=\bar{w}_{1} Q_{m}\left(\frac{f-r_{1}}{\bar{r}_{1}} ; \boldsymbol{H}_{2}^{(1)}\right)+w_{1}=\bar{w}_{1} Q_{m}\left(\frac{\alpha_{2}\left(f-r_{1}\right)}{\bar{r}_{1}} ; \boldsymbol{H}_{3}^{(1)}\right)+w_{1} \text {. }
$$

We claim that, for $0 \leq f<1$,

$$
Q_{m+1}\left(f ; \boldsymbol{H}_{2}\right) \geq Q_{m+1}\left(\alpha_{2} f ; \boldsymbol{H}_{3}\right) .
$$

If $f<\min \left\{1, r_{1} / \alpha_{2}\right\}$ (implying that $\alpha_{2} f<r_{1} \leq 1$ ), we have, by (2),

$$
Q_{m+1}\left(\alpha_{2} f ; \boldsymbol{H}_{3}\right)=w_{1} Q_{m}\left(\frac{\alpha_{2} f}{r_{1}} ; \boldsymbol{H}_{3}^{(1)}\right),
$$

which either equals $Q_{m+1}\left(f ; \boldsymbol{H}_{2}\right)$ if $f<r_{1} \leq \min \left\{1, r_{1} / \alpha_{2}\right\}$ (by (5)) or is bounded from above by $w_{1}$ and, hence, by $Q_{m+1}\left(f ; \boldsymbol{H}_{2}\right)$ if $r_{1} \leq f<\min \left\{1, r_{1} / \alpha_{2}\right\}$ (by (6)). If $r_{1} / \alpha_{2} \leq f<1$ (which is not vacuous only when $r_{1} / \alpha_{2}<1$ ) then we have

$$
\begin{aligned}
Q_{m+1}\left(\alpha_{2} f ; \boldsymbol{H}_{3}\right) & =\bar{w}_{1} Q_{m}\left(\frac{\alpha_{2} f-r_{1}}{\bar{r}_{1}} ; \boldsymbol{H}_{3}^{(1)}\right)+w_{1} \\
& \leq \bar{w}_{1} Q_{m}\left(\frac{\alpha_{2}\left(f-r_{1}\right)}{\bar{r}_{1}} ; \boldsymbol{H}_{3}^{(1)}\right)+w_{1} \\
& =Q_{m+1}\left(f ; \boldsymbol{H}_{2}\right),
\end{aligned}
$$

where the inequality follows from the monotonicity of $Q_{m}$ (Lemma 1) and the last equality follows from (6). This proves (7), which together with statement (S3) and Lemma 2 yields, for $0 \leq y \leq f<1$ and $f+y \bar{r}_{1} / r_{1}<1$,

$$
\begin{aligned}
Q_{m+1}\left(f ; \boldsymbol{H}_{2}\right) & \geq Q_{m+1}\left(\alpha_{2} f ; \boldsymbol{H}_{3}\right) \\
& \geq \bar{w}_{1} Q_{m}\left(\alpha_{2} f-\alpha_{2} y ; \boldsymbol{H}_{3}^{(1)}\right)+w_{1} Q_{m}\left(\alpha_{2} f+\frac{\alpha_{2} y \bar{r}_{1}}{r_{1}} ; \boldsymbol{H}_{3}^{(1)}\right) \\
& =\bar{w}_{1} Q_{m}\left(f-y ; \boldsymbol{H}_{2}^{(1)}\right)+w_{1} Q_{m}\left(f+\frac{y \bar{r}_{1}}{r_{1}} ; \boldsymbol{H}_{2}^{(1)}\right) .
\end{aligned}
$$


By Lemma 3, for $0 \leq y \leq f<1$ and $f+y \bar{r}_{1} / r_{1} \geq 1$ (implying that $(1-f) r_{1} / \bar{r}_{1} \leq y \leq$ $f<1)$,

$$
Q_{m+1}\left(f ; \boldsymbol{H}_{2}\right) \geq \bar{w}_{1} Q_{m}\left(f-y ; \boldsymbol{H}_{2}^{(1)}\right)+w_{1} Q_{m}\left(f+\frac{y \bar{r}_{1}}{r_{1}} ; \boldsymbol{H}_{2}^{(1)}\right) .
$$

This proves that (4) holds for $n=m, \boldsymbol{H}=\boldsymbol{H}_{2}$, and all $0 \leq y \leq f<1$.

(iii) (S4) $\Rightarrow$ (S3): To prove that (4) holds for $0 \leq y \leq f<1, n=m$, and $\boldsymbol{H}=\boldsymbol{H}_{3}$, it suffices (by Lemma 3) to consider $0 \leq y<(1-f) r_{1} / \bar{r}_{1}$. We now assume that $0 \leq y<B(f):=$ $\min \left\{f,(1-f) r_{1} / \bar{r}_{1}\right\}$ (the 'bold stake' on the first play with respect to both $\boldsymbol{H}_{3}$ and $\boldsymbol{H}_{4}$ ). We have, by (2), $\boldsymbol{H}_{3}^{(1)}=\boldsymbol{H}_{4}^{(1)}, w_{1} \geq w_{2}$, Lemma 1 , and $y<B(f)$,

$$
\begin{aligned}
Q_{m+1}( & \left.f ; \boldsymbol{H}_{3}\right)-Q_{m+1}\left(f ; \boldsymbol{H}_{4}\right) \\
= & \left(\bar{w}_{1} Q_{m}\left(f-B(f) ; \boldsymbol{H}_{3}^{(1)}\right)+w_{1} Q_{m}\left(f+\frac{B(f) \bar{r}_{1}}{r_{1}} ; \boldsymbol{H}_{3}^{(1)}\right)\right) \\
& -\left(\bar{w}_{2} Q_{m}\left(f-B(f) ; \boldsymbol{H}_{4}^{(1)}\right)+w_{2} Q_{m}\left(f+\frac{B(f) \bar{r}_{1}}{r_{1}} ; \boldsymbol{H}_{4}^{(1)}\right)\right) \\
= & \left(w_{1}-w_{2}\right)\left(Q_{m}\left(f+\frac{B(f) \bar{r}_{1}}{r_{1}} ; \boldsymbol{H}_{3}^{(1)}\right)-Q_{m}\left(f-B(f) ; \boldsymbol{H}_{3}^{(1)}\right)\right) \\
\geq & \left(w_{1}-w_{2}\right)\left(Q_{m}\left(f+\frac{y \bar{r}_{1}}{r_{1}} ; \boldsymbol{H}_{3}^{(1)}\right)-Q_{m}\left(f-y ; \boldsymbol{H}_{3}^{(1)}\right)\right) .
\end{aligned}
$$

Then it follows from statement (S4) and $\boldsymbol{H}_{3}^{(1)}=\boldsymbol{H}_{4}^{(1)}$ that

$$
\begin{aligned}
Q_{m+1} & \left(f ; \boldsymbol{H}_{3}\right)-\left(\bar{w}_{1} Q_{m}\left(f-y ; \boldsymbol{H}_{3}^{(1)}\right)+w_{1} Q_{m}\left(f+\frac{y \bar{r}_{1}}{r_{1}} ; \boldsymbol{H}_{3}^{(1)}\right)\right) \\
& \geq Q_{m+1}\left(f ; \boldsymbol{H}_{4}\right)-\left(\bar{w}_{2} Q_{m}\left(f-y ; \boldsymbol{H}_{3}^{(1)}\right)+w_{2} Q_{m}\left(f+\frac{y \bar{r}_{1}}{r_{1}} ; \boldsymbol{H}_{3}^{(1)}\right)\right) \\
& \geq 0,
\end{aligned}
$$

proving (4) for $0 \leq y<B(f), n=m$, and $\boldsymbol{H}=\boldsymbol{H}_{3}$.

(iv) (S5) $\Rightarrow$ (S4): To prove that (4) holds for $0 \leq y \leq f<1, n=m$, and $\boldsymbol{H}=\boldsymbol{H}_{4}$, it suffices (by Lemma 3 ) to consider $0 \leq y<(1-f) r_{1} / \bar{r}_{1}$, implying that

$$
f+\frac{y \bar{r}_{1}}{r_{1}}<1 \text {. }
$$

Let $y^{\prime}=r_{2} y / r_{1}$ and $f^{\prime}=f+\left(r_{2}-r_{1}\right) y / r_{1}$. It is easily checked that

$$
0 \leq y^{\prime} \leq f^{\prime}, \quad f^{\prime}-y^{\prime}=f-y, \quad f^{\prime}+\frac{y^{\prime} \bar{r}_{2}}{r_{2}}=f+\frac{y \bar{r}_{1}}{r_{1}} .
$$

Note that, by (8), $f^{\prime} \leq f+\left(1-r_{1}\right) y / r_{1}<1$. Then, by statement (S5), (9), and $\boldsymbol{H}_{4}^{(1)}=\boldsymbol{H}_{5}^{(1)}$,

$$
\begin{aligned}
Q_{m+1}\left(f^{\prime} ; \boldsymbol{H}_{5}\right) & \geq \bar{w}_{2} Q_{m}\left(f^{\prime}-y^{\prime} ; \boldsymbol{H}_{5}^{(1)}\right)+w_{2} Q_{m}\left(f^{\prime}+\frac{y^{\prime} \bar{r}_{2}}{r_{2}} ; \boldsymbol{H}_{5}^{(1)}\right) \\
& =\bar{w}_{2} Q_{m}\left(f-y ; \boldsymbol{H}_{4}^{(1)}\right)+w_{2} Q_{m}\left(f+\frac{y \bar{r}_{1}}{r_{1}} ; \boldsymbol{H}_{4}^{(1)}\right) .
\end{aligned}
$$


It now suffices to show that

$$
Q_{m+1}\left(f^{\prime} ; \boldsymbol{H}_{5}\right) \leq Q_{m+1}\left(f ; \boldsymbol{H}_{4}\right) .
$$

For $0 \leq f<r_{1}$, we have

$$
f^{\prime} \leq f+\frac{\left(r_{2}-r_{1}\right) f}{r_{1}}=\frac{r_{2} f}{r_{1}}<r_{2},
$$

so by (2), Lemma 1 , and $\boldsymbol{H}_{4}^{(1)}=\boldsymbol{H}_{5}^{(1)}$,

$$
Q_{m+1}\left(f ; \boldsymbol{H}_{4}\right)=w_{2} Q_{m}\left(\frac{f}{r_{1}} ; \boldsymbol{H}_{4}^{(1)}\right) \geq w_{2} Q_{m}\left(\frac{f^{\prime}}{r_{2}} ; \boldsymbol{H}_{4}^{(1)}\right)=Q_{m+1}\left(f^{\prime} ; \boldsymbol{H}_{5}\right),
$$

proving (10). For $r_{1} \leq f<1$, we have, by (2),

$$
Q_{m+1}\left(f ; \boldsymbol{H}_{4}\right)=\bar{w}_{2} Q_{m}\left(\frac{f-r_{1}}{\bar{r}_{1}} ; \boldsymbol{H}_{4}^{(1)}\right)+w_{2} .
$$

If $f^{\prime}<r_{2}$ then, by (2) and (11), $Q_{m+1}\left(f^{\prime} ; \boldsymbol{H}_{5}\right) \leq w_{2} \leq Q_{m+1}\left(f ; \boldsymbol{H}_{4}\right)$, proving (10). If $r_{2} \leq f^{\prime}<1$ then

$$
\begin{aligned}
Q_{m+1}\left(f^{\prime} ; \boldsymbol{H}_{5}\right) & =\bar{w}_{2} Q_{m}\left(\frac{f^{\prime}-r_{2}}{\bar{r}_{2}} ; \boldsymbol{H}_{5}^{(1)}\right)+w_{2} \\
& \leq \bar{w}_{2} Q_{m}\left(\frac{f-r_{1}}{\bar{r}_{1}} ; \boldsymbol{H}_{5}^{(1)}\right)+w_{2} \\
& =Q_{m+1}\left(f ; \boldsymbol{H}_{4}\right),
\end{aligned}
$$

proving (10), where the inequality follows from Lemma 1 and the fact that

$$
\frac{f-r_{1}}{\bar{r}_{1}}-\frac{f^{\prime}-r_{2}}{\bar{r}_{2}}=\frac{r_{2}-r_{1}}{\bar{r}_{1} \bar{r}_{2}}\left(1-\left(f+y \frac{\bar{r}_{1}}{r_{1}}\right)\right) \geq 0
$$

(by (8)). The proof is complete.

Proof of Proposition 1. We proceed by induction on $n$. Clearly, (4) holds for $n=0$, all $0 \leq y \leq f<1$, and all $\boldsymbol{H}=(\boldsymbol{r}, \boldsymbol{w}, \boldsymbol{\alpha})$ satisfying (1). Suppose that (4) holds for all $n<m$ $(m \geq 1)$, all $0 \leq y \leq f<1$, and all $\boldsymbol{H}=(\boldsymbol{r}, \boldsymbol{w}, \boldsymbol{\alpha})$ satisfying (1). We now prove that, for $0 \leq y \leq f<1$ and $\boldsymbol{H}$ satisfying (1),

$$
Q_{m+1}(f ; \boldsymbol{H}) \geq \bar{w}_{1} Q_{m}\left(\alpha_{1}(f-y) ; \boldsymbol{H}^{(1)}\right)+w_{1} Q_{m}\left(\alpha_{1}\left(f+\frac{y \bar{r}_{1}}{r_{1}}\right) ; \boldsymbol{H}^{(1)}\right) .
$$

If $\left(1-r_{1}\right) w_{1} \alpha_{1}=0$ then $Q_{k}(f ; \boldsymbol{H})=0$ for $0 \leq f<1$ and $k \geq 1$, so (12) holds trivially. If $\left(1-r_{2}\right) w_{2} \alpha_{2}=0$ then $Q_{m+1}(f ; \boldsymbol{H})=Q_{1}(f ; \boldsymbol{H})$ and $Q_{m}\left(f ; \boldsymbol{H}^{(1)}\right)=Q_{0}\left(f ; \boldsymbol{H}^{(1)}\right)=$ $\mathbf{1}_{[1, \infty)}(f)$, so (12) reduces to (4) with $n=0$, which holds trivially. Hence, it remains to consider those $\boldsymbol{H}$ satisfying (1) and $0<\alpha_{1} \leq 1,0<\alpha_{2} \leq 1,0<w_{2} \leq w_{1}$, and $r_{1} \leq r_{2}<1$. In view of Lemma 4 , it suffices to prove (12) for $0 \leq y \leq f<1$ and $\boldsymbol{H}$ satisfying (1) and $\alpha_{1}=\alpha_{2}=1,0<w_{1}=w_{2}=: w$, and $1>r_{1}=r_{2}=: r$, i.e. $\boldsymbol{\alpha}=\left(1,1, \alpha_{3}, \alpha_{4}, \ldots\right), \boldsymbol{w}=$ $\left(w, w, w_{3}, w_{4}, \ldots\right)$, and $\boldsymbol{r}=\left(r, r, r_{3}, r_{4}, \ldots\right)$. 
We now establish (12) for $0=y<f<1$, i.e. $Q_{m+1}(f ; \boldsymbol{H}) \geq Q_{m}\left(f ; \boldsymbol{H}^{(1)}\right)$, noting that $\boldsymbol{H}$ satisfies $\alpha_{1}=\alpha_{2}=1,0<w_{1}=w_{2}=w \leq \frac{1}{2}$, and $\frac{1}{2} \leq r_{1}=r_{2}=r<1$. For $0<f<r$, we have, by (2) and the induction hypothesis,

$$
\begin{aligned}
Q_{m+1}(f ; \boldsymbol{H}) & =w Q_{m}\left(\frac{f}{r} ; \boldsymbol{H}^{(1)}\right) \\
& \geq w\left(\bar{w} Q_{m-1}\left(\frac{f}{r}-0 ; \boldsymbol{H}^{(2)}\right)+w Q_{m-1}\left(\frac{f}{r}+0 \cdot \frac{\bar{r}}{r} ; \boldsymbol{H}^{(2)}\right)\right) \\
& =w Q_{m-1}\left(\frac{f}{r} ; \boldsymbol{H}^{(2)}\right) \\
& =Q_{m}\left(f ; \boldsymbol{H}^{(1)}\right),
\end{aligned}
$$

and, for $r \leq f<1$,

$$
\begin{aligned}
Q_{m+1}(f ; \boldsymbol{H}) & =\bar{w} Q_{m}\left(\frac{f-r}{\bar{r}} ; \boldsymbol{H}^{(1)}\right)+w \\
& \geq \bar{w}\left(\bar{w} Q_{m-1}\left(\frac{f-r}{\bar{r}}-0 ; \boldsymbol{H}^{(2)}\right)+w Q_{m-1}\left(\frac{f-r}{\bar{r}}+0 \cdot \frac{\bar{r}}{r} ; \boldsymbol{H}^{(2)}\right)\right)+w \\
& =\bar{w} Q_{m-1}\left(\frac{f-r}{\bar{r}} ; \boldsymbol{H}^{(2)}\right)+w \\
& =Q_{m}\left(f ; \boldsymbol{H}^{(1)}\right)
\end{aligned}
$$

proving (12) for $0=y<f<1$. In view of Lemma 3, it remains to prove (12) for

$$
0<y<\min \left\{f, \frac{(1-f) r}{\bar{r}}\right\}=: B(f) .
$$

Since (13) implies that

$$
0<f-y<f<f+\frac{y \bar{r}}{r}<1,
$$

it suffices to consider the following four cases separately. (The rest of the proof follows the one in [15, Lemma 1] closely.)

Case (i): $f-y<f<f+y \bar{r} / r \leq r$. In this case $B(f)=f$. By (2) and the induction hypothesis,

$$
\begin{aligned}
Q_{m+1}(f ; \boldsymbol{H}) & =w Q_{m}\left(\frac{f}{r} ; \boldsymbol{H}^{(1)}\right) \\
& \geq w\left(\bar{w} Q_{m-1}\left(\frac{f}{r}-\frac{y}{r} ; \boldsymbol{H}^{(2)}\right)+w Q_{m-1}\left(\frac{f}{r}+\frac{y}{r} \frac{\bar{r}}{r} ; \boldsymbol{H}^{(2)}\right)\right) \\
& =\bar{w}\left(w Q_{m-1}\left(\frac{f-y}{r} ; \boldsymbol{H}^{(2)}\right)\right)+w\left(w Q_{m-1}\left(\frac{f+y \bar{r} / r}{r} ; \boldsymbol{H}^{(2)}\right)\right) \\
& =\bar{w} Q_{m}\left(f-y ; \boldsymbol{H}^{(1)}\right)+w Q_{m}\left(f+\frac{y \bar{r}}{r} ; \boldsymbol{H}^{(1)}\right)
\end{aligned}
$$

proving (12). 
Case (ii): $r \leq f-y<f<f+y \bar{r} / r$. Let $f^{\prime}=(f-r) / \bar{r}$ and $y^{\prime}=y / \bar{r}$. Note that $0<y^{\prime} \leq f^{\prime}<1$ (since $r \leq f-y$ implies that $y^{\prime} \leq f^{\prime}$ ) and that $f-B(f)=f^{\prime}$ (since $B(f)=(1-f) r / \bar{r})$. By (2) and the induction hypothesis,

$$
\begin{aligned}
Q_{m+1}(f ; \boldsymbol{H})= & \bar{w} Q_{m}\left(f^{\prime} ; \boldsymbol{H}^{(1)}\right)+w \\
\geq & \bar{w}\left(\bar{w} Q_{m-1}\left(f^{\prime}-y^{\prime} ; \boldsymbol{H}^{(2)}\right)+w Q_{m-1}\left(f^{\prime}+\frac{y^{\prime} \bar{r}}{r} ; \boldsymbol{H}^{(2)}\right)\right)+w \\
= & \bar{w}\left(\bar{w} Q_{m-1}\left(f^{\prime}-y^{\prime} ; \boldsymbol{H}^{(2)}\right)+w\right) \\
& +w\left(\bar{w} Q_{m-1}\left(\frac{f+y \bar{r} / r-r}{\bar{r}} ; \boldsymbol{H}^{(2)}\right)+w\right) \\
= & \bar{w} Q_{m}\left(f-y ; \boldsymbol{H}^{(1)}\right)+w Q_{m}\left(f+\frac{y \bar{r}}{r} ; \boldsymbol{H}^{(1)}\right),
\end{aligned}
$$

proving (12), where the second equality follows from the fact that

$$
f^{\prime}+\frac{y^{\prime} \bar{r}}{r}=\frac{f+y \bar{r} / r-r}{\bar{r}}
$$

and the last equality follows from (2) and the fact that

$$
f^{\prime}-y^{\prime}=\frac{f-y-r}{\bar{r}} .
$$

Case (iii): $f-y<f<r<f+y \bar{r} / r$. Since $y<f=B(f)$, we have

$$
r<f+\frac{y \bar{r}}{r}<\frac{f}{r}<1 .
$$

So, by (2),

$$
\begin{aligned}
& Q_{m+1}(f ; \boldsymbol{H})=w Q_{m}\left(\frac{f}{r} ; \boldsymbol{H}^{(1)}\right) \\
&=w\left(\bar{w} Q_{m-1}\left(\frac{f / r-r}{\bar{r}} ; \boldsymbol{H}^{(2)}\right)+w\right) \\
&=\bar{w}\left(w Q_{m-1}\left(\frac{f / \bar{r}-r^{2} / \bar{r}}{r} ; \boldsymbol{H}^{(2)}\right)\right)+w^{2} \\
&=\bar{w} Q_{m}\left(\frac{f}{\bar{r}}-\frac{r^{2}}{\bar{r}} ; \boldsymbol{H}^{(1)}\right)+w^{2}, \\
& Q_{m}(f-\left.y ; \boldsymbol{H}^{(1)}\right)=w Q_{m-1}\left(\frac{f-y}{r} ; \boldsymbol{H}^{(2)}\right), \\
& Q_{m}\left(f+y \frac{\bar{r}}{r} ; \boldsymbol{H}^{(1)}\right)=\bar{w} Q_{m-1}\left(\frac{f+y \bar{r} / r-r}{\bar{r}} ; \boldsymbol{H}^{(2)}\right)+w .
\end{aligned}
$$

It follows that (12) is equivalent to

$$
Q_{m}\left(\frac{f}{\bar{r}}-\frac{r^{2}}{\bar{r}} ; \boldsymbol{H}^{(1)}\right) \geq w Q_{m-1}\left(\frac{f-y}{r} ; \boldsymbol{H}^{(2)}\right)+w Q_{m-1}\left(\frac{f+y \bar{r} / r-r}{\bar{r}} ; \boldsymbol{H}^{(2)}\right) .
$$


Note that here $f / \bar{r}-r^{2} / \bar{r},(f-y) / r$, and $(f+y \bar{r} / r-r) / \bar{r}$, the arguments in $Q_{m}$ and $Q_{m-1}$, all lie in the set $[0,1)$. It suffices to consider the following two subcases.

Subcase (iii.1):

$$
\frac{f-y}{r} \geq \frac{f+y \bar{r} / r-r}{\bar{r}} \geq 0 .
$$

Let $f^{\prime}=f^{\prime}(f, y)$ and $y^{\prime}=y^{\prime}(f, y)$ be as defined in [15, Lemma 4] (with $\left.\alpha=1\right)$. Then $0 \leq y^{\prime} \leq f^{\prime}$. Since $r \geq \frac{1}{2}$ and $f+y \bar{r} / r>r$, we have

$$
\frac{f}{\bar{r}}-\frac{r^{2}}{\bar{r}}-f^{\prime}=\left(\frac{r}{\bar{r}}-1\right)\left(f+\frac{y \bar{r}}{r}-r\right) \geq 0 .
$$

It follows from Lemma 1, [15, Lemma 4], the induction hypothesis, and $w \leq \frac{1}{2}$ that

$$
\begin{aligned}
Q_{m}\left(\frac{f}{\bar{r}}-\frac{r^{2}}{\bar{r}} ; \boldsymbol{H}^{(1)}\right) & \geq Q_{m}\left(f^{\prime} ; \boldsymbol{H}^{(1)}\right) \\
& \geq \bar{w} Q_{m-1}\left(f^{\prime}-y^{\prime} ; \boldsymbol{H}^{(2)}\right)+w Q_{m-1}\left(f^{\prime}+\frac{y^{\prime} \bar{r}}{r} ; \boldsymbol{H}^{(2)}\right) \\
& \geq w Q_{m-1}\left(\frac{f+y \bar{r} / r-r}{\bar{r}} ; \boldsymbol{H}^{(2)}\right)+w Q_{m-1}\left(\frac{f-y}{r} ; \boldsymbol{H}^{(2)}\right),
\end{aligned}
$$

proving (15) and, hence, (12).

Subcase (iii.2):

$$
\frac{f+y \bar{r} / r-r}{\bar{r}} \geq \frac{f-y}{r} \geq 0 .
$$

Let $f^{\prime}=f^{\prime}(f, y)$ and $y^{\prime}=y^{\prime}(f, y)$ be as defined in [15, Lemma 5] (with $\left.\alpha=1\right)$. Then $0 \leq y^{\prime} \leq f^{\prime}$. Since $r \geq \frac{1}{2}$ and $f>y$, we have

$$
\frac{f}{\bar{r}}-\frac{r^{2}}{\bar{r}}-f^{\prime}=\frac{(r-\bar{r})(f-y)}{r} \geq 0 .
$$

By Lemma 1, [15, Lemma 5], the induction hypothesis, and $w \leq \frac{1}{2}$,

$$
\begin{aligned}
Q_{m}\left(\frac{f}{\bar{r}}-\frac{r^{2}}{\bar{r}} ; \boldsymbol{H}^{(1)}\right) & \geq Q_{m}\left(f^{\prime} ; \boldsymbol{H}^{(1)}\right) \\
& \geq \bar{w} Q_{m-1}\left(f^{\prime}-y^{\prime} ; \boldsymbol{H}^{(2)}\right)+w Q_{m-1}\left(f^{\prime}+\frac{y^{\prime} \bar{r}}{r} ; \boldsymbol{H}^{(2)}\right) \\
& \geq w Q_{m-1}\left(\frac{f-y}{r} ; \boldsymbol{H}^{(2)}\right)+w Q_{m-1}\left(\frac{f+y \bar{r} / r-r}{\bar{r}} ; \boldsymbol{H}^{(2)}\right),
\end{aligned}
$$

proving (15) and, hence, (12). 
Case (iv): $f-y<r \leq f<f+y \bar{r} / r$. In this case, by (13), $y<B(f)=(1-f) r / \bar{r}$, so

$$
r>f-y>f-B(f)=\frac{f-r}{\bar{r}} .
$$

Then, by (2),

$$
\begin{gathered}
Q_{m+1}(f ; \boldsymbol{H})=\bar{w} Q_{m}\left(\frac{f-r}{\bar{r}} ; \boldsymbol{H}^{(1)}\right)+w \\
=\bar{w} w Q_{m-1}\left(\frac{f-r}{\bar{r}} \frac{1}{r} ; \boldsymbol{H}^{(2)}\right)+w \\
Q_{m}\left(f-y ; \boldsymbol{H}^{(1)}\right)=w Q_{m-1}\left(\frac{f-y}{r} ; \boldsymbol{H}^{(2)}\right), \\
Q_{m}\left(f+y \frac{\bar{r}}{r} ; \boldsymbol{H}^{(1)}\right)=\bar{w} Q_{m-1}\left(\frac{f+y \bar{r} / r-r}{\bar{r}} ; \boldsymbol{H}^{(2)}\right)+w .
\end{gathered}
$$

It follows that (12) is equivalent to

$$
\begin{aligned}
\bar{w}+ & \bar{w} Q_{m-1}\left(\frac{f-r}{\bar{r}} \frac{1}{r} ; \boldsymbol{H}^{(2)}\right) \\
& \geq \bar{w} Q_{m-1}\left(\frac{f-y}{r} ; \boldsymbol{H}^{(2)}\right)+\bar{w} Q_{m-1}\left(\frac{f+y \bar{r} / r-r}{\bar{r}} ; \boldsymbol{H}^{(2)}\right) .
\end{aligned}
$$

Note that $(f / r-1) / \bar{r},(f-y) / r$, and $(f+y \bar{r} / r-r) / \bar{r}$, the arguments in $Q_{m-1}$, all lie in the set $[0,1)$. Since $r \leq f / r-1+r<1$, we have, by (2),

$$
Q_{m}\left(\frac{f}{r}-1+r ; \boldsymbol{H}^{(1)}\right)=\bar{w} Q_{m-1}\left(\frac{f / r-1}{\bar{r}} ; \boldsymbol{H}^{(2)}\right)+w .
$$

Thus, (16) is equivalent to

$$
\begin{aligned}
& 1-2 w+Q_{m}\left(\frac{f}{r}-1+r ; \boldsymbol{H}^{(1)}\right) \\
& \geq \bar{w} Q_{m-1}\left(\frac{f-y}{r} ; \boldsymbol{H}^{(2)}\right)+\bar{w} Q_{m-1}\left(\frac{f+y \bar{r} / r-r}{\bar{r}} ; \boldsymbol{H}^{(2)}\right) .
\end{aligned}
$$

It remains to prove (17) for the following two subcases.

Subcase (iv.1):

$$
\frac{f-y}{r} \geq \frac{f+y \bar{r} / r-r}{\bar{r}} \geq 0 .
$$

Let $f^{\prime}=f^{\prime}(f, y)$ and $y^{\prime}=y^{\prime}(f, y)$ be as defined in [15, Lemma 4] (with $\left.\alpha=1\right)$. Then $0 \leq y^{\prime} \leq f^{\prime}$. Since $r \geq \frac{1}{2}$ and $f-y<r$,

$$
\frac{f}{r}-1+r-f^{\prime}=\frac{(2 r-1)(r-(f-y))}{r} \geq 0 .
$$


By Lemma 1, [15, Lemma 4], the induction hypothesis, and $w \leq \frac{1}{2}$,

$$
\begin{aligned}
Q_{m}( & \left.\frac{f}{r}-1+r ; \boldsymbol{H}^{(1)}\right) \\
& \geq Q_{m}\left(f^{\prime} ; \boldsymbol{H}^{(1)}\right) \\
& \geq \bar{w} Q_{m-1}\left(f^{\prime}-y^{\prime} ; \boldsymbol{H}^{(2)}\right)+w Q_{m-1}\left(f^{\prime}+\frac{y^{\prime} \bar{r}}{r} ; \boldsymbol{H}^{(2)}\right) \\
& =\bar{w} Q_{m-1}\left(\frac{f+y \bar{r} / r-r}{\bar{r}} ; \boldsymbol{H}^{(2)}\right)+w Q_{m-1}\left(\frac{f-y}{r} ; \boldsymbol{H}^{(2)}\right) \\
& \geq \bar{w} Q_{m-1}\left(\frac{f+y \bar{r} / r-r}{\bar{r}} ; \boldsymbol{H}^{(2)}\right)+\bar{w} Q_{m-1}\left(\frac{f-y}{r} ; \boldsymbol{H}^{(2)}\right)-(1-2 w),
\end{aligned}
$$

from which (17) (and, hence, (12) and (16)) follows.

Subcase (iv.2):

$$
\frac{f+y \bar{r} / r-r}{\bar{r}} \geq \frac{f-y}{r} \geq 0 .
$$

Let $f^{\prime}=f^{\prime}(f, y)$ and $y^{\prime}=y^{\prime}(f, y)$ be as defined in [15, Lemma 5] (with $\left.\alpha=1\right)$. Then $0 \leq y^{\prime} \leq f^{\prime}$. Since $\frac{1}{2} \leq r<1$ and $f+y \bar{r} / r<1$ (by (14)),

$$
\frac{f}{r}-1+r-f^{\prime}=\frac{(2 r-1)(1-(f+y \bar{r} / r))}{1-r} \geq 0 .
$$

By Lemma 1, [15, Lemma 5], the induction hypothesis, and $w \leq \frac{1}{2}$,

$$
\begin{aligned}
Q_{m}( & \left.\frac{f}{r}-1+r ; \boldsymbol{H}^{(1)}\right) \\
& \geq Q_{m}\left(f^{\prime} ; \boldsymbol{H}^{(1)}\right) \\
& \geq \bar{w} Q_{m-1}\left(f^{\prime}-y^{\prime} ; \boldsymbol{H}^{(2)}\right)+w Q_{m-1}\left(f^{\prime}+\frac{y^{\prime} \bar{r}}{r} ; \boldsymbol{H}^{(2)}\right) \\
& =\bar{w} Q_{m-1}\left(\frac{f-y}{r} ; \boldsymbol{H}^{(2)}\right)+w Q_{m-1}\left(\frac{f+y \bar{r} / r-r}{\bar{r}} ; \boldsymbol{H}^{(2)}\right) \\
& \geq \bar{w} Q_{m-1}\left(\frac{f-y}{r} ; \boldsymbol{H}^{(2)}\right)+\bar{w} Q_{m-1}\left(\frac{f+y \bar{r} / r-r}{\bar{r}} ; \boldsymbol{H}^{(2)}\right)-(1-2 w),
\end{aligned}
$$

from which (17) (and, hence, (12) and (16)) follows. This completes the proof.

\section{Acknowledgements}

The first author gratefully acknowledges support from the National Science Council of Taiwan under grants NSC 094-2118-M-001-019 and NSC 096-2628-M-001-023. The second author is a postdoc fellow supported by the National Science Council of Taiwan under grant NSC 096-2811-M-001-065. 


\section{References}

[1] Chen, M.-R. and Hsiau, S.-R. (2006). Two-person red-and-black games with bet-dependent win probability functions. J. Appl. Prob. 43, 905-915.

[2] Chen, R. (1978). Subfair 'red-and-black' in the presence of inflation. Z. Wahrscheinlichkeitsth. 42, $293-301$.

[3] Chen, R. And Zame, A. (1979). On discounted subfair primitive casino. Z. Wahrscheinlichkeitsth. 49, 257-266.

[4] Chen, R., Shepp, L. A., Yao, Y.-C. and Zhang C.-H. (2005). On optimality of bold play for primitive casinos in the presence of inflation. J. Appl. Prob. 42, 121-137.

[5] Dubins, L. E. And Savage, L. J. (1976). Inequalities for Stochastic Processes (How to Gamble if You Must). Dover, New York.

[6] Grigorescu, I., Chen, R. And Shepp, L. (2007). Optimal strategy for the Vardi casino with interest payments. J. Appl. Prob. 44, 199-211.

[7] Klugman, S. (1977). Discounted and rapid subfair red-and-black. Ann. Statist. 5, 734-745.

[8] Maitra, A. P. and Sudderth, W. D. (1996). Discrete Gambling and Stochastic Games (Appl. Math. New York 32). Springer, New York.

[9] Pontiggia, L. (2005). Two-person red-and-black with bet-dependent win probability. Adv. Appl. Prob. 37, 75-89.

[10] Pontiggia, L. (2007). Nonconstant sum red-and-black games with bet-dependent win probability function. J. Appl. Prob. 44, 547-553.

[11] Schweinsberg, J. (2005). Improving on bold play when the gambler is restricted. J. Appl. Prob. 42, 321-333.

[12] Shaked, M and Shanthikumar, J. G. (2007). Stochastic Orders. Springer, New York.

[13] Shepr, L. A. (2006). Bold play and the optimal policy for Vardi's casino. In Random Walk, Sequential Analysis and Related Topics, eds A. C. Hsiung et al., World Scientific, Singapore, pp. 150-156.

[14] Sudderth, W. D. and Weerasinghe, A. (1989). Controlling a process to a goal in finite time. Math. Operat. Res. 14, 400-409.

[15] Yao, Y.-C. (2007). On optimality of bold play for discounted Dubins-Savage gambling problems with limited playing times. J. Appl. Prob. 44, 212-225.

[16] Yao, Y.-C. (2007). Explicit optimal strategy for the Vardi casino with limited playing time. J. Appl. Prob. 44, 831-837. 